\title{
Article
}

Subscriber access provided by University of East Anglia Library

\section{Exposing the Interplay Between Enzyme Turnover, Protein Dynamics and the Membrane Environment in Monoamine Oxidase B}

Hannah B. L. Jones, Rory M Crean, Anna Mullen, Emanuele Kendrick, Steven D. Bull, Stephen A. Wells, David Ross Carbery, Fraser MacMillan, Marc W. van der Kamp, and Christopher Roland Pudney

Biochemistry, Just Accepted Manuscript • DOI: 10.1021/acs.biochem.9b00213 • Publication Date (Web): 09 Apr 2019

Downloaded from http://pubs.acs.org on April 16, 2019

\section{Just Accepted}

"Just Accepted" manuscripts have been peer-reviewed and accepted for publication. They are posted online prior to technical editing, formatting for publication and author proofing. The American Chemical Society provides "Just Accepted" as a service to the research community to expedite the dissemination of scientific material as soon as possible after acceptance. "Just Accepted" manuscripts appear in full in PDF format accompanied by an HTML abstract. "Just Accepted" manuscripts have been fully peer reviewed, but should not be considered the official version of record. They are citable by the Digital Object Identifier (DOI®). "Just Accepted" is an optional service offered to authors. Therefore, the "Just Accepted" Web site may not include all articles that will be published in the journal. After a manuscript is technically edited and formatted, it will be removed from the "Just Accepted" Web site and published as an ASAP article. Note that technical editing may introduce minor changes to the manuscript text and/or graphics which could affect content, and all legal disclaimers and ethical guidelines that apply to the journal pertain. ACS cannot be held responsible for errors or consequences arising from the use of information contained in these "Just Accepted" manuscripts. 


\title{
Exposing the Interplay Between Enzyme Turnover, Protein Dynamics and the Membrane Environment in Monoamine Oxidase B
}

\author{
Hannah B. L. Jones ${ }^{\dagger \|}$, Rory M. Crean $\#+\|$, Anna Mullen§, Emanuele G. Kendrick ${ }^{\dagger}$, Steven D Bull ${ }^{\perp}$, Stephen A. Wells ${ }^{\ddagger}$, David \\ R. Carbery ${ }^{\wedge}$, Fraser MacMillan§, Marc W. Van der Kamp*\%, Christopher R. Pudney*广†.
}

${ }^{\dagger}$ Department of Biology and Biochemistry, ${ }^{\Delta}$ Department of Chemistry, ${ }^{\star}$ Department of Chemical Engineering University of Bath, "Doctoral Training Centre in Sustainable Chemical Technologies, 'Centre for Therapeutic Innovation, University of Bath, Bath BA2 7AY, United Kingdom.

\%School of Biochemistry, University of Bristol, Biomedical Sciences building, University Walk, Bristol BS8 1TD, United Kingdom.

§School of Chemistry, University of East Anglia, Norwich Research Park, Norwich NR4 7TJ, United Kingdom.

\begin{abstract}
There is an increasing realization that structure-based drug design may show improved success by understanding the ensemble of conformations and sub-states accessible to an enzyme and how the environment affects this ensemble. Human monoamine oxidase B (MAO-B) catalyzes the oxidation of amines and is inhibited for the treatment of both Parkinson's disease and depression. Despite its clinical importance, its catalytic mechanism remains unclear and routes to drugging this target would be valuable. Evidence of a radical in either the transition state or resting state of MAO-B is present throughout the literature, and is suggested to be a flavin semiquinone, a tyrosyl radical or both. Here we see evidence of a resting state flavin semiquinone, via absorption redox studies and electron paramagnetic resonance, suggesting that the anionic semiquinone is biologically relevant. Based on enzyme kinetic studies, enzyme variants and molecular dynamics simulations we find evidence for the importance of the membrane environment in mediating the activity of MAO-B and that this mediation is related to the protein dynamics of MAO-B. Further, our MD simulations identify a hitherto undescribed entrance for substrate binding, membrane modulated substrate access, and indications for half-site reactivity: only one active site is accessible to binding at a time. Our study combines both experimental and computational evidence to illustrate the subtle interplay between enzyme activity, protein dynamics and the immediate membrane environment. Understanding key biomedical enzymes to this level of detail will be crucial to inform strategies (and binding sites) for rational drug design for these targets.
\end{abstract}

\section{KEYWORDS EPR, Molecular Dynamics, Monoamine Oxidase B, semiquinone, enzyme, flavin, membrane}

Introduction. Human monoamine Oxidase B (MAO-B) catalyzes the oxidative deamination of amines, by electron transfer, via its flavin adenine dinucleotide (FAD) cofactor (Scheme 1). ${ }^{1}$ It is located in the outer mitochondrial membrane, ${ }^{2}$ as a dimer, with bipartite substrate binding and active site cavities. ${ }^{1}$ MAO-B is the target of treatment for both depression and Parkinson's disease, with inhibitors of the enzyme first being approved as pharmaceuticals in the 1960 's. ${ }^{3,4}$

Despite the important medical applications associated with MAO-B its chemical mechanism remains unclear and there is debate over the role of MAO-B conformational change and protein dynamics. Reduction of the FAD has been shown to proceed by a tunneling mechanism via primary $\left(1^{\circ}\right)$ and secondary $\left(2^{\circ}\right)$ kinetic isotope effect (KIE) studies. ${ }^{5}$ These studies have suggested that MAO-B catalysis is not linked to fast (pico/nanosecond) dynamics. ${ }^{5,6} \mathrm{MAO}-\mathrm{B}$ catalysis has been investigated via experimental and computational studies, with at least seven different proposed mechanisms, including polar nucleophilic, ${ }^{7}$ radical ${ }^{8}$ direct hydride transfer ${ }^{9-11}$ and two step hydride transfer ${ }^{12}$ (Scheme S1).

Although the direct single electron transfer (SET) radical mechanism (Scheme S1) has previously been discounted experimentally ${ }^{13}$ and by quantum mechanics/molecular mechanics $(\mathrm{QM} / \mathrm{MM}),{ }^{14}$ a separate radical mechanism has been proposed by Murray et al. ${ }^{15}$ This was established via a model small molecule reaction that mimics MAO-B to provide evidence for a neutral semiquinone flavin that can be formed aerobically. The authors suggest that a neutral semiquinone flavin is the reactive species for the oxidation of benzylamine (BZA). ${ }^{15}$ This led to the hypothesis of a radical mechanism whereby MAO-B forms a neutral semiquinone flavin via a proximal tyrosine radical (Y398). The presence of a stable anionic semiquinone flavin and tyrosyl radical intermediate are also reported in MAO-A and MAO-N. . $^{16,17}$

In our hands, we find spectroscopic evidence for a stable

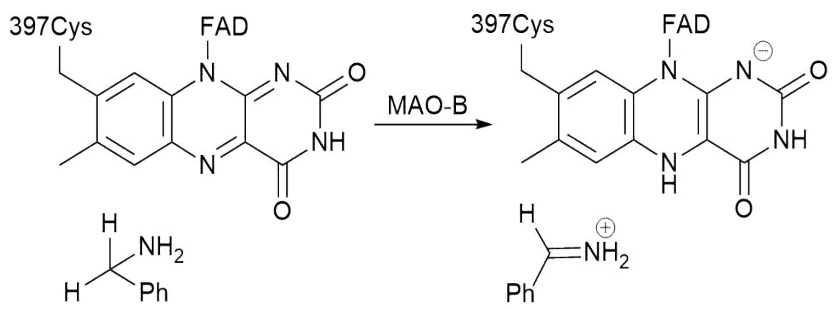
semiquinone in resting state MAO-B (vida infra). Here, MAO-B

Scheme 1. General reaction catalyzed by MAO-B. 

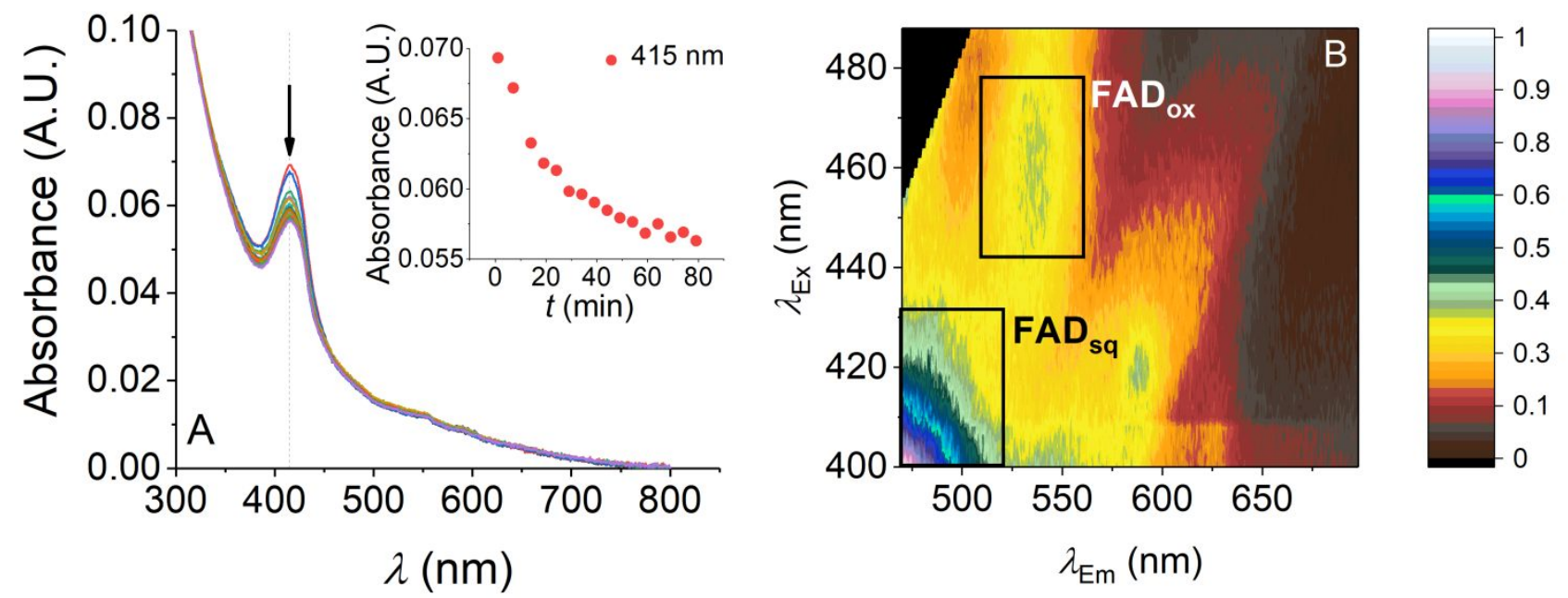

Figure 1. A, The absorbance spectra of MAO-B recorded at various time points after treatment with BZA. Inset, the effect on the $415 \mathrm{~nm}$ 
peak over time. B, Fluorescence excitation/emission matrix resolves oxidized and semiquinone flavin states (highlighted in solid black boxes). Scale bar is relative intensity Conditions: $50 \mathrm{mM}$ HEPES $0.5 \% \mathrm{w} / \mathrm{v}$ Triton X-100, $20^{\circ} \mathrm{C}$. For absorption experiments $40 \mathrm{mM}$ BZA, anaerobic conditions as Materials and Methods.

was expressed and purified in Pichia pastoris as outlined by Newton-Vinson et al. ${ }^{18}$ This protocol attributes observations of oxidized/semiquinone MAO-B FAD to reactive oxygen species (ROS) that form upon the disruption of the mitochondrial membrane, which they observe upon purification from bovine liver, but not from P. pastoris. ${ }^{18}$

Previous observation of a stable anionic semiquinone FAD in MAO-B ${ }^{19,20}$ led to a hypothesis of half-site reactivity. This mechanism posits that one monomer of the MAO-B dimer is inaccessible to oxygen and substrate, resulting in the formation of the stable semiquinone species, whilst the other contains oxidized FAD. Electrons are then shuttled to the semiquinone species, allowing for the oxidation of the reduced FAD upon turnover..$^{20}$ The authors suggest this might arise from electron shuttling between specific amino acids. Potentially, such a mechanism might require significant conformational change associated with turnover in each monomer. Indeed, conformational changes have been found to be associated with MAO-B turnover, with a molecular dynamics (MD) study demonstrating the potential for the membrane to regulate access to the active site entrance via two gating loops (residues 85-119 and 155-165). ${ }^{21}$ Other MD studies have been carried out on MAO-B, both with and without a membrane environment, with the focus on identifying or improving inhibitors for MAO-B ${ }^{22-}$ ${ }^{26}$, or ascertaining how MAO-B binds to the membrane. ${ }^{27}$

Herein, we find evidence for a resting-state anionic semiquinone, and through kinetic and computational studies, evidence for conformationally controlled enzyme activity at each MAO-B monomer. Crucially, we find that the membrane environment exposes novel substrate/product channels that could be potential new drug targets. We thereby link the membrane environment, substrate binding and MAO-B conformational change to enzyme turnover.

\section{Materials and Methods}

Unless otherwise stated, all reagents were obtained from SigmaAldrich.

MAO-B expression and purification. MAO-B was expressed and purified following the purification protocol by NewtonVinson et al. ${ }^{18}$ Small variations from the protocol include shake flask fermentation instead of bioreactor fermentation, with BMMY (buffered methanol-complex medium) media instead of MM (minimal methanol) media for induction, storage of cell pellets in a buffer with protease inhibitor tablets instead of PMSF, suspension of pellet in $100 \mathrm{ml}$ of breaking buffer instead of $1 \mathrm{~L}$, and cell breakage of $30 \mathrm{~s}$ on $30 \mathrm{~s}$ off $\mathrm{x} 10$ sonication in addition to bead beating. The purification was completed after MAO-B was passed over a DEAE-sepharose FF column, achieving satisfactory purity. The additional polymer partition step detailed by Newton-Vinson et al. ${ }^{18}$ did not change the state of the semiquinone MAO-B species seen here, with additional purity achieved by size exclusion chromatography if necessary. MAO-B concentration was determined spectroscopically using $\varepsilon_{415}=11000 \mathrm{M}^{-1} \mathrm{~cm}^{-1}$. $^{28}$
Nanodisc preparation. Nanodiscs were prepared following methods by McDowall et al., ${ }^{29}$ suspended in $50 \mathrm{mM}$ HEPES, $\mathrm{pH}$ 7.5. and incubated with MAO-B in a 10x molar excess, for 2 hours prior to experiments.

Enzyme assays. MAO-B was transferred from Triton X-100 containing buffer to reduced Triton X-100 or nanodisc containing buffer using detergent removal spin columns (Thermo Scientific Pierce). Steady-state MAO-B kinetic measurements were carried out using a $1 \mathrm{ml}$ quartz cuvette and a UV/ Vis spectro-photometer (Agilent Cary $60 \mathrm{UV}-\mathrm{Vis}$ spectrometer) in $50 \mathrm{mM}$ HEPES ( $\mathrm{pH} 7.5$ ), containing $0.5 \%$ (w/v) reduced Triton X-100 or SMA 1- $\alpha$-Phosphatidylcholine nanodiscs. Enzyme activity was measured by following the formation of benzaldehyde using $\varepsilon_{250}=12,800 \mathrm{M}^{-1} \mathrm{~cm}^{-1}$ for BZA, ${ }^{13}$ and 4-hydroxyquinoline using $\varepsilon_{316}=12,300 \mathrm{M}^{-1} \mathrm{~cm}^{-1}$ for $\mathrm{KYN}^{30}$ For each condition substrate dependences were monitored at $40{ }^{\circ} \mathrm{C}$; the data fitted well to Michaelis-Menten kinetics (Figure S2). Temperature dependences were carried out from $20^{\circ} \mathrm{C}-45^{\circ} \mathrm{C}$ at $5^{\circ} \mathrm{C}$ intervals using initial velocity measurements at substrate concentrations maintained above $10 \mathrm{x} K_{\mathrm{m}}$ to ensure saturation. The data were fitted to Eq 2 as described using OriginPro 2017.

Redox assays. These experiments were performed anaerobically, all buffer was purged with nitrogen and samples were prepared in an anaerobic box. Glucose and glucose oxidase were added to maintain anaerobic conditions.

EPR. Measurements were performed using WT and Y398F variant MAO-B in $50 \mathrm{mM}$ HEPES $0.5 \%$ Triton, $\mathrm{pH} 7.5$, flashfrozen in liquid nitrogen in suprasil quartz sample tubes. $\mathrm{X}$ band cw-EPR spectra were recorded on a Bruker eleXsys E500 spectrometer using a standard rectangular Bruker EPR cavity (ER4102T) equipped with an Oxford helium cryostat (ESR900). Experimental parameters: microwave power, $0.2 \mathrm{~mW}$; field modulation amplitude, $5 \mathrm{G}$; field modulation frequency, $100 \mathrm{kHz}$; measuring time $10 \mathrm{~s}$; temperature $16 \mathrm{~K}$. Qband cw-EPR spectra were performed on a Bruker eleXsys E560 spectrometer using a ER 5106QT-W1 resonator equipped with a home-built ARS cryogen-free cryostat (data not shown). Spectral simulations were performed using the Matlab-based Easyspin package. ${ }^{31}$

Computational materials and methods. The X-ray crystal structure of MAO-B in complex with 6-hydroxy-N-propargyl$1(\mathrm{R})$-aminoindan $(1 \mathrm{~S} 3 \mathrm{E})^{32}$ was used as the starting point for all MD simulations. The missing C-terminal residues (502 - 520 Chain A and 497 - 520 Chain B) which form the remainder of the transmembrane helix were built using Avogadro ${ }^{33}$ (assuming the standard backbone dihedral angles of an $\alpha$-helix). MAO-B was then inserted into a lipid bilayer comprised of a 4:3 ratio of palmitoyloleoylphosphatidylcholine (POPC) and palmitoyloleoyl-phosphatidylethanolamine (POPE) using CHARMM-GUI. ${ }^{34}$ This composition has been used in prior bilayer MD simulations of MAO-B, and was chosen to represent the composition of the outer mitochondrial membrane. ${ }^{21,27,35}$ BZA was placed in the active site by alignment with the crystal structure of MAO-B in complex with nitrobenzylamine $\left(2 \mathrm{C} 70,{ }^{36} \mathrm{C} \alpha \mathrm{RMSD} 0.118 \AA\right.$ to $1 \mathrm{~S} 3 \mathrm{E}$; the 


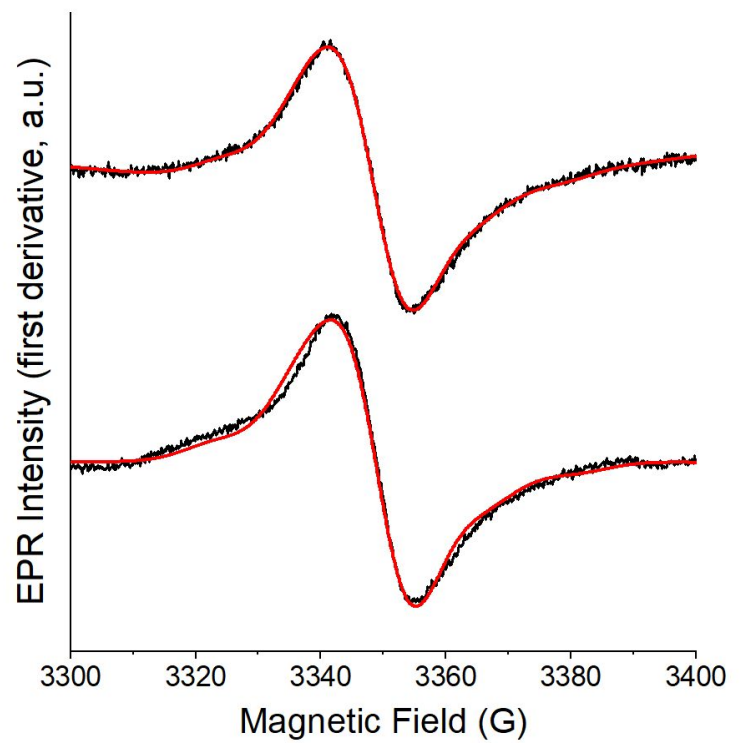

latter was used due to its higher resolution). All simulations of BZA were performed with the amino group in its neutral form, as this is widely believed to be the catalytically competent state of BZA. $8,14,37$ Titratable residues were simulated in their standard protonation states, based on calculations with PropKa 3.0. ${ }^{38}$ MolProbity was used to determine the optimum tautomerisation states of every His residue, and any required Asn/Gln side chain flips, based on optimizing the hydrogen bonding network. ${ }^{39}$ Histidines 91, 115 and 452 were singly protonated on their $\mathrm{N} \delta 1$, with all others singly protonated on their N\&2. The system was then solvated such that there was no protein or lipid atom within $20 \AA$ of the edge of the periodic box along the $\mathrm{z}$-coordinate (bilayer normal). The total number of atoms for each system simulated was approximately 90,000.

Periodic boundary simulations were performed with Amber16, using the CHARMM36 force field to describe protein ${ }^{40}$ and lipid $^{41}$ atoms, and TIP3P for water. Parameters for FAD in its oxidized form and BZA in its neutral form were taken from Abad et al. ${ }^{14}$ Following minimization, heating and equilibration (see SI Materials and Methods), production MD simulations were run in the $\mathrm{NpT}$ ensemble at $310 \mathrm{~K}$, with semi-isotropic coupling to a Monte Carlo barostat. Temperature was regulated using Langevin dynamics with a collision frequency of $1 \mathrm{ps}^{-1}$. A time step of 2 fs was applied with the covalent bonds to hydrogen constrained by the SHAKE algorithm. A $12 \AA$ nonbonded cut-off was applied with a force switch smoothing function from 10 to $12 \AA$. Long range electrostatics were evaluated with the particle mesh Ewald method. ${ }^{42}$ For $w t$ MAOB simulations, a total of 9 simulations were performed for 150 ns each, with the first $50 \mathrm{~ns}$ of simulation used to equilibrate the protein and bilayer (see Figure S5). The $\mathrm{BZA}_{2}$ run in which a BZA escapes the active site cavity was extended for a further 50 ns. Enzyme variant simulations (W184F, F402V, E466Y) were performed for $3 \times 100 \mathrm{~ns}$ each in the BZA1 state (see SI Materials and Methods for further details). Coordinates were saved every $10 \mathrm{ps}$ for further analysis.Routine analysis of trajectories was performed using CPPTRAJ ${ }^{43}$, from the AmberTools suite. Area per lipid calculations were performed with GridMAT-MD, ${ }^{44}$ using a grid resolution of $200 \times 200$ points for each measurement. Tunnel analysis was performed with Caver 3.0. ${ }^{45}$ All settings were kept default apart from bottleneck radius (1.5 $\AA$ ).

\section{Results and Discussion}

Evidence for a resting-state semiquinone in MAO- $B$. The presence of purified MAO-B was established by SDS-PAGE (Figure $\mathrm{S} 1$; essentially a single band via size exclusion chromatography) and via electrospray ionization quadrupole time-of-flight (ESI-Q TOF) mass spectrometry in combination with the MASCOT server. ${ }^{46}$ The absorption spectrum of purified MAO-B is shown in Figure 1A. The spectra share characteristics of an anionic semiquinone FAD (FAD--) with an absorption feature at $\sim 415 \mathrm{~nm} .{ }^{47}$ The preparation protocol of MAO-B was the same as that used by Newton-Vinson et al., ${ }^{18}$ with small differences outlined in the Materials and Methods. Multiple preparations were completed, including the final polymer partition step outlined by Newton-Vinson et al. and without EDTA present, with the $\sim 415 \mathrm{~nm}$ spectral feature consistently present. The effect of incubation with BZA, under anaerobic conditions, on the MAO-B absorption feature at $\sim 415$ $\mathrm{nm}$ was monitored over time (Figure 1A inset). From Figure $1 \mathrm{~A}$ inset, we find a decrease in absorption at $415 \mathrm{~nm}$ with respect to time on incubation with BZA, suggesting the $415 \mathrm{~nm}$ spectral feature is redox sensitive with a natural MAO-B substrate.

We note that the absorption spectrum shown in Figure 1A lacks a defined feature around $\sim 450 \mathrm{~nm}$ where one would expect oxidized flavin as well as spectral features at $\sim 475 \mathrm{~nm}$ that would also characterize a putative anionic semiquinone. We do not have a clear explanation for the lack of these features and the complexity of the absorption spectrum; the protein is purified to homogeneity, it is identified as MAO-B by mass spectrometry and is catalytically active with the natural substrates (Figure 1A inset, Figure S2 and as described below). The electronic environment around both flavins is highly complex, with a large number of proximal Tyr residues (Y60, 398 and 435 positioned $3.1,3.3$ and $3.5 \AA$ from the alloxan moiety, respectively). Potentially, this gives rise to a complex absorption spectrum arising from different electronic environments for a sub-set of active site conformational states. This hypothesis requires MAO-B to be able to explore different conformational states and we consider this in more detail below. Given the complexity of the absorption spectrum, we turned to fluorescence and electron paramagnetic resonance (EPR) spectroscopy to provide more specific evidence for the oxidation state(s) of the MAO-B flavin.

Figure 2. X-band cw-EPR spectra of WT (top, black) and Y398F (bottom, black) MAO-B, with their respective fitted simulations (red). EPR microwave frequency $=9.3916 \mathrm{GHz}$ (WT) \& $9.3926 \mathrm{GHz}(\mathrm{Y} 398 \mathrm{~F})$, microwave power $=0.2 \mathrm{~mW}$, modulation amplitude $=0.5 \mathrm{mT}$, temperature $=16 \mathrm{KPreviously}$, MAO-B steady-state fluorescence spectroscopy has demonstrated that there are two different chromophores present in resting state MAO-B. ${ }^{50}$ The authors concluded that these two different species were consistent with oxidized and semiquinone flavin. To establish if we similarly have both oxidized and seminquinoid flavin present (which is not obvious from the absorption spectrum), we have monitored the fluorescence excitation-emission matrix (Figure 1B). Similar to the previous report, the spectra resolve at least two different emission peaks suggesting the presence of two different oxidation states of the flavin with $\lambda_{\mathrm{Ex}} \sim 400-420$ and $460 \mathrm{~nm}$. Notably, the excitation/emission profile at $\lambda_{\mathrm{Ex}} \sim 460$ and $\lambda_{\mathrm{Em}} \sim$ $540 \mathrm{~nm}$ (Figure 1B) indicates the presence of oxidized flavin. 
The $\sim 415 \mathrm{~nm}$ absorption feature could be potentially attributed to a tyrosyl radical, which has a characteristic absorbance peak at $410 \mathrm{~nm},{ }^{48}$ or a mixture of both an FAD semiquinone and tyrosyl radical, as seen as intermediates in MAO-A. ${ }^{16,17}$ Murray et al. postulated that the reactive semiquinone FAD was formed by a proximal tyrosyl radical (Y398). However, the UV-vis absorption spectrum of Y398F MAO-B also shows the spectral feature at $\sim 415 \mathrm{~nm}$ (Figure S3A), indicating that this feature is not attributable to a Y398 tyrosyl radical. The Y398F variant also shows a slight increase in $K_{\mathrm{m}}$ (Figure S4), similar in magnitude to previously reported changes in $K_{\mathrm{m}}$ for $\mathrm{Y} 398 \mathrm{~F} .{ }^{49}$

A stringent approach to identifying the nature of the flavin oxidation state is EPR. The EPR X-band spectrum of the wild type MAO-B ( $w t \mathrm{MAO}-\mathrm{B})$ clearly indicates that the signal arises from a semiquinone radical (Figure 2), in agreement with the UV-Vis and fluorescence spectroscopy (Figure 1). The measured spectra lack the defining features which would identify the signal as arising from a tyrosyl radical: the typical 'wings' or 'shoulders' around the central signal at around $\mathrm{g}=2$ 16,51 at X-band (Figure 2), and increased g-value anisotropy at higher frequency (and therefore resonant field), i.e. Q-band (data not shown). Furthermore, the signal persists in the Y398F variant, confirming that it is not caused by the proximal tyrosine. Further, computational simulation and fitting of the experimental X-band data of the WT and Y398F MAO-B (Table 1) suggests that the semiquinone radical species is anionic; the hyperfine environment of a neutral semiquinone radical would contain a contribution from an additional hydrogen atom. ${ }^{20,52}$ This is not the case for the signals seen in the X-band spectra.

Previous studies have illustrated the importance of the membrane environment in mediating the normal enzymatic activity of MAO-B. ${ }^{21}$ To probe if the putative semiquinoid species (inferred from spectroscopic studies above) was also stably present in the membrane environment, we have conducted spectroscopic studies in an artificial membrane

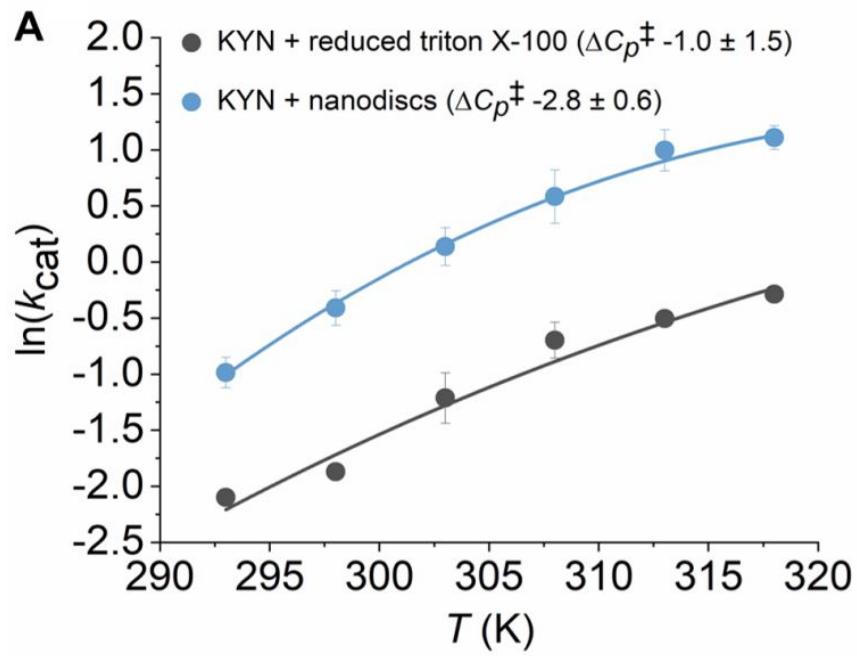

Table 1. Spectral Parameters of EPR Data Extracted by Simulation and Fitting

\begin{tabular}{ll|ll}
\hline & & WT MAO- & Y398F MAO- \\
& & B & B \\
\hline g-tensor & $\mathrm{g}_{\mathrm{x}}$ & 2.00444 & 2.00444 \\
& $\mathrm{~g}_{\mathrm{y}}$ & 2.00429 & 2.00429 \\
& $\mathrm{~g}_{\mathrm{z}}$ & 2.00191 & 2.00191 \\
& $\mathrm{~g}_{\text {iso }}$ & 2.00355 & 2.00355 \\
\hline $\mathbf{A}\left({ }^{\mathbf{1 4}} \mathbf{N}\right) \mathbf{( M H z )}$ & $\mathrm{A}_{\|}$ & 39.3 & 38.4 \\
& $\mathrm{~A}_{\text {[ }}$ & 0 & 0 \\
\hline $\mathbf{A}\left({ }^{\mathbf{1 4}} \mathbf{N}\right)(\mathbf{M H z})$ & $\mathrm{A}_{\|}$ & 34.1 & 40.1 \\
& $\mathrm{~A}_{\square}$ & 0 & 0 \\
\hline Linewidth (mT) & & 1.1 & 1.1 \\
\hline RMSD & & 0.0255 & 0.0440 \\
\hline
\end{tabular}

environment using $1-\alpha$-phosphatidylcholine styrene maleic acid co-polymer (SMA) nanodiscs, prepared as reported previously. ${ }^{29}$ We find that the absorption feature at $\sim 415 \mathrm{~nm}$ is present in both reduced Triton X-100 and nanodisc environments (Figure S3A), implying that the putative anionic semiquinone is not an artifact of the buffer system used, and that its presence is not affected by the specific membrane environment used. It is clear from these data (Figures 1 and 2) that the FAD of resting state MAO-B is able to stably occupy oxidation states other than fully oxidized FAD. Evidence for this is recurring in the literature, demonstrated by Raman ${ }^{19}$, fluorescence, ${ }^{50}$ and $\mathrm{EPR}^{18,20}$ spectroscopy. We suggest that these different observations could be rationalized by an equilibrium of energetically similar conformational states, which allow differently stable oxidation states. Indeed, below we provide evidence that shows MAO-B is able to sample a range of conformational states.

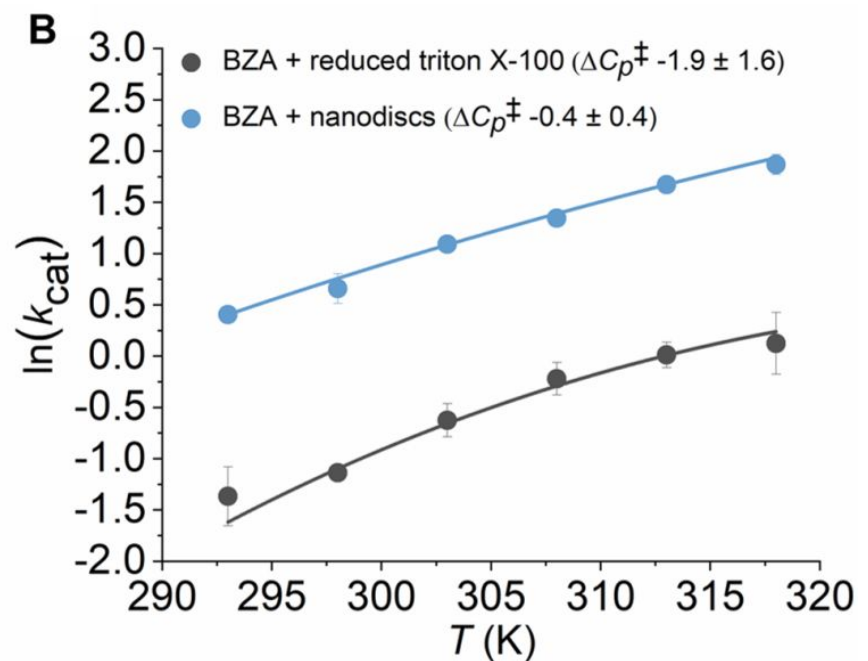

Figure 3. The temperature dependence of MAO-B turnover with KYN (A) and BZA (B) with reduced Triton and nanodisc environments, fit to the MMRT equation. Conditions, $50 \mathrm{mM}$ HEPES $0.5 \% \mathrm{w} / \mathrm{v}$ reduced Triton X-100 $\mathrm{pH} 7.5$. Reduced Triton X-100: $1.5 \mathrm{mM}$ BZA, 0.75 mM KYN. Nanodiscs: 3 mM BZA, $0.66 \mathrm{mM} \mathrm{KYN.}$

Influence of the membrane environment on MAO-B turnover. To assess how/if the membrane environment affects enzyme turnover, we measured MAO-B turnover with both BZA and kynuramine dihydrobromide $(\mathrm{KYN})$ at a range of temperatures. We monitor enzyme turnover based on the absorption features of benzaldehyde product formation at $250 \mathrm{~nm}$ for BZA, ${ }^{13}$ and 
4-hydroxyquinoline product formation at $316 \mathrm{~nm}$ for $\mathrm{KYN} .^{30}$ This assay notionally primarily reflects the rate of reduction of the flavin. ${ }^{13}$ Our temperature dependence studies allow us to analyze not just the observed rate of enzyme turnover but also the thermodynamics of the system. The temperaturedependence of the observed rate is shown in Figure 3.

There has been a recent move to fitting enzyme temperature dependence data to physical models that allow for curvature in the associated plots. Such models often provide a more realistic fit to experimental data. We fit the MAO-B temperature dependence data to a model that incorporates the heat capacity of catalysis $\left(\Delta C_{P}^{\ddagger}\right)(\mathrm{Eq} 2)$ into the Eyring equation $(\mathrm{Eq} 1){ }^{53}$

$k=\left(\frac{k_{\mathrm{B}} T}{h}\right) e^{\frac{\Delta S^{\ddagger}}{R}} e^{-\frac{\Delta H^{\ddagger}}{R T}}$

$\ln k=\ln \frac{k_{B} T}{h}-\left[\frac{\Delta H_{T_{0}^{*}}^{\ddagger}+\Delta C_{P}^{\ddagger}\left(T-T_{0}\right)}{R T}\right]+\left[\frac{\Delta S_{T_{0}^{*}}^{\ddagger}+\Delta C_{P}^{\ddagger}\left(\ln T-\ln T_{0}\right)}{R}\right]$

Eq 2

Where $\Delta H^{\ddagger}$ is the change in enthalpy and $\Delta S^{\ddagger}$ is the change in entropy between the ground and transition state of the reaction at an arbitrary reference temperature $\left(T_{0}\right)$. This model has recently gained traction in studying enzyme temperature dependencies. ${ }^{54-57}$ In the absence of other confounding factors, $\Delta C_{P}^{\ddagger}$ quantifies the temperature dependence of $\Delta H^{\star}$ and $\Delta S^{\ddagger}$ and reflects the difference in the distribution and frequency of vibrational modes between the ground state and transition state. ${ }^{53,58}$ We have recently suggested that $\Delta C_{P}^{\ddagger}$ can be used as a proxy for the changes in these vibrational modes during enzyme where the membrane environment may not alter the tertiary structure of the enzyme, but potentially alters protein fluctuations, which have previously been proposed to affect small molecule binding to the active site. ${ }^{21}$

From Figure 3 and Table $\mathrm{S} 1$, we find $\Delta C_{P}^{\ddagger}$ to be the same within error for both KYN and BZA substrates when in a reduced Triton X-100 environment. However, when in nanodiscs, the difference in $\Delta C_{P}^{\ddagger}$ for the different substrates is $\Delta \Delta C_{P}^{\ddagger}=2.4 \pm$ $1.0 \mathrm{~kJ} \mathrm{~mol}^{-1}$. The $\Delta C_{P}^{\ddagger}$ increases in magnitude from reduced Triton to nanodiscs with KYN, and decreases with BZA (Figure 3 ). These data suggest that the difference in conformational fluctuations in the reactant and transition states is different in a more native membrane environment and for different substrates. Moreover, we find a significant difference in the observed rate of enzyme turnover in the nanodiscs $(\sim 5$ times faster). These data therefore provide experimental evidence of the notion that the membrane environment has a role in 'tuning' the molecular dynamics of MAO-B. However, given we observe a retention of the putative anionic flavin semiquinone, we would suggest the membrane does not affect the chemical mechanism of enzyme turnover per se.

Computational evidence for a new entrance to the MAO-B active site mediated by the protein-membrane interaction. To obtain detailed insight into the role of the membrane environment on protein dynamics, we performed MD simulations of the MAO-B dimer embedded in a phospholipid membrane. We explore (i) the influence of the phospholipid membrane in modulating substrate/inhibitor binding, (ii) the accessibility of small molecules to the active site of MAO-B, and (iii) the potential for half-site reactivity, as discussed above.

turnover and thus relates to some aspect of the protein's molecular dynamics. ${ }^{55}$ This is relevant in the present study
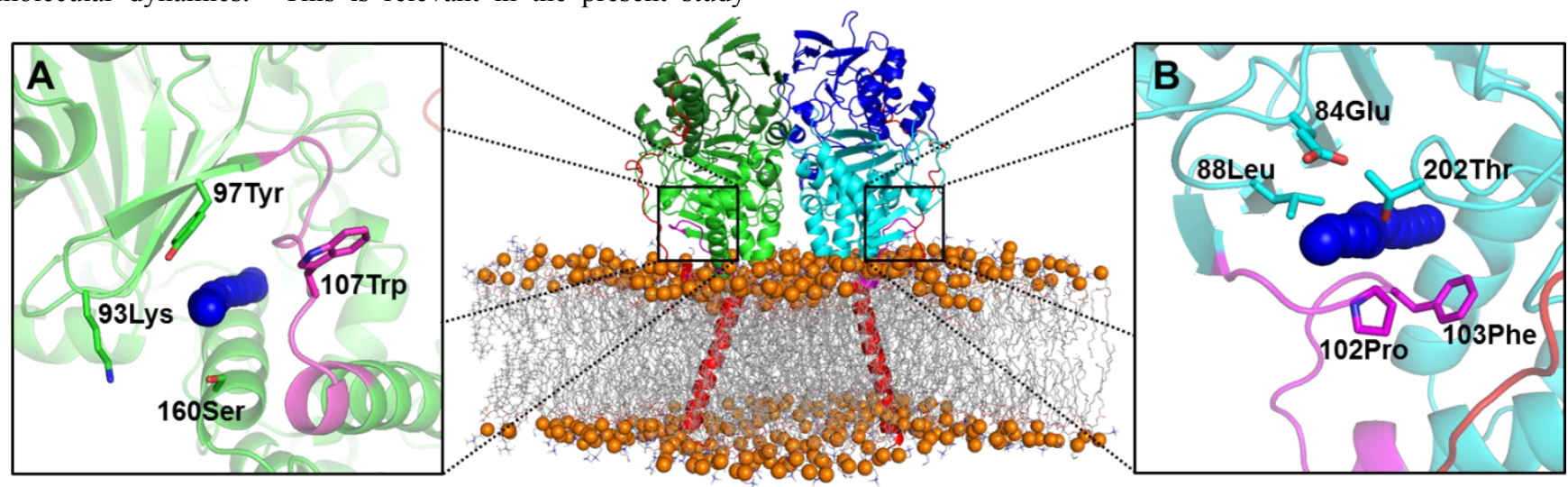

Figure 4. MAO-B in POPC/POPE membrane environment. The substrate binding area is shown in light green and light blue (residues 80 210, 286-390) for each monomer. The active site area is shown in dark green and dark blue (residues 4-79, 211-285, and 391-452). The Cterminal tail and transmembrane helix are shown in red (residues 453-520). The binding site gating loop is shown in magenta (residues 99112). Images A and B inset denote the two main entrances (or tunnels) to the binding site (denoted Entrance A and Entrance B throughout the manuscript), with a representative tunnel (navy spheres) shown for both entrances. Key residues which describe the location of the entrance are shown as sticks and labelled. Entrance B is newly identified here, Entrance A has been previously described. ${ }^{21}$

MD simulations of MAO-B in complex with FAD in a POPC/POPE lipid bilayer (similar to the native environment in the mitochondrial membrane $)^{35}$ were performed in triplicate for 150 ns in 3 different states: no BZA present $\left(\mathrm{BZA}_{0}\right)$, one active site occupied with BZA (BZA $)$, and both active sites occupied with BZA $\left(\mathrm{BZA}_{2}\right)$. Protein $\mathrm{C} \alpha$ RMSD and area per lipid head groups (Figure $\mathrm{S} 5$ ) indicated equilibration of both the protein and membrane after $50 \mathrm{~ns}$ of production MD. The analysis described below is therefore from 50 to $150 \mathrm{~ns}$.

Prior simulations of $\mathrm{BZA}_{0}-\mathrm{MAO}-\mathrm{B}$ in a bilayer established that MAO-B ligand binding site access is modulated by the membrane. ${ }^{21}$ We investigate this further using longer simulations (150 ns vs. $50 \mathrm{~ns}$ ) and with substrate (BZA) bound. 
To measure possible access to the substrate cavity of MAO-B via the membrane, we quantify the occurrence and features of tunnels in our simulations using Caver 3.0. ${ }^{45}$ Tunnels identified are grouped into clusters, allowing for the quantification of various characteristics, such as the frequency of occurrence and smallest width (bottleneck radius), as used here. ${ }^{59}$ This tool has previously been used to identify tunnels for ligand-induced protein flexibility analysis, ${ }^{60}$ to rationalize change in mechanism and kinetics of an enzyme upon a point mutation, ${ }^{61}$ and to identify a tunnel to the FAD moiety in MAO-A. ${ }^{62}$

Two main possible entrances for ligands into the MAO-B active site are found at either side of the gating loop residues 99 to 112 (Figure 4). Entrance A (Figure 4A) is accessed via the membrane and its opening has previously been observed. ${ }^{21}$ Briefly, a $\pi-\pi$ stacking interaction between Tyr97 and Trp107 is lost as Trp107 buries into the aliphatic lipid tails of the bilayer, establishing an additional interaction of MAO-B with the phospholipid bilayer.

Entrance B (Figure 4B) is solvent accessible, and its opening is controlled by the conformation of three loop regions ( 81 to 88 , 99 to 112 and 198 to 208). To the best of our knowledge, its opening has not yet been observed through protein crystallography or simulation. To provide a qualitative description of the open and closed conformations of this entrance, we have performed clustering analysis on the entrance loop residues (see Materials and Methods). Opening of Entrance B (Figure S6) can be described by: 1) loop 81-88 separates from loop 199-206, breaking a number of transiently
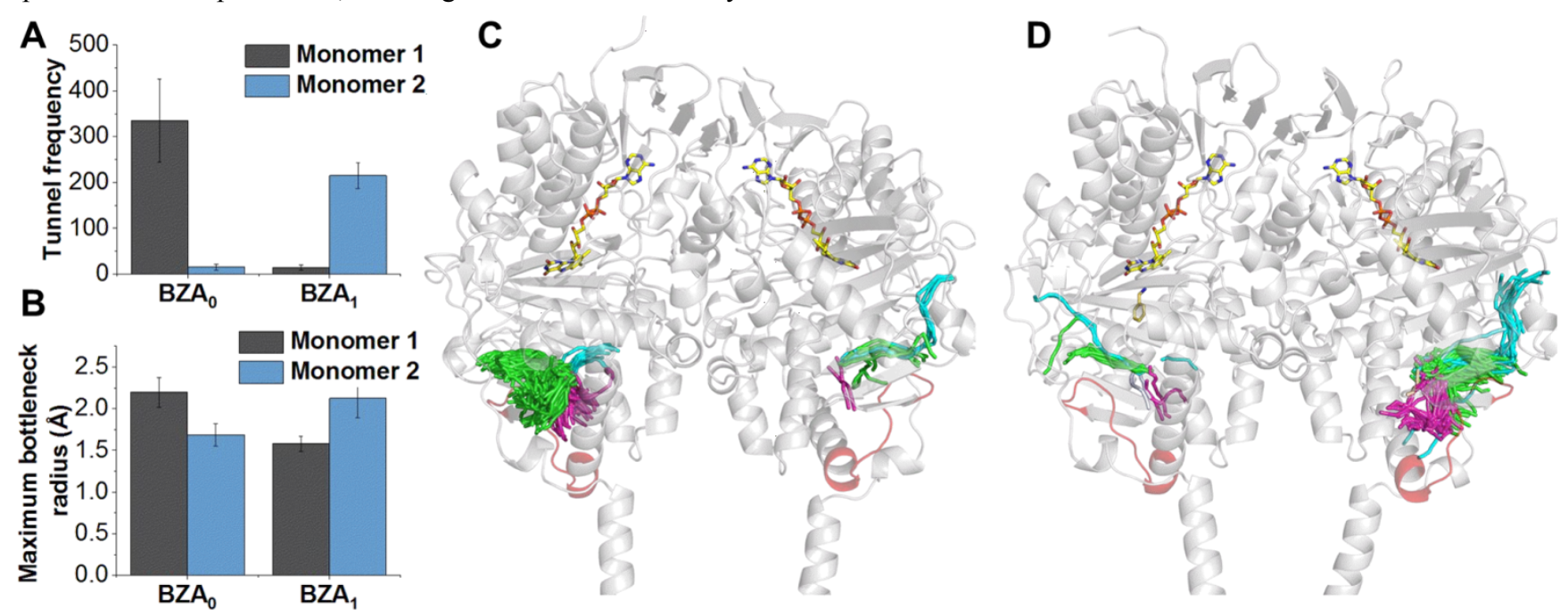

Figure 5. A, The summed tunnel frequency from the substrate binding site of MAO-B to solvent, identified by Caver. ${ }^{45} \mathrm{BZA}_{0}-\mathrm{No}_{\mathrm{BZA}}$ is bound in either monomer. $\mathrm{BZA}_{1}-\mathrm{BZA}$ is bound only in monomer 1 . All are from triplicate MD runs. B, The average maximum bottleneck $(\AA)$ from the substrate binding site of MAO-B to the solvent, as identified by Caver. $\mathbf{C}+\mathbf{D}$, All tunnels identified by Caver over all three MD simulation repeats for $\mathrm{BZA}_{0}(\mathbf{C})$ and $\mathrm{BZA}_{1}(\mathbf{D})$. FAD and BZA (when present) are shown as yellow sticks. Tunnels are colored according to entrance/exit pathway used, with magenta and green representing Entrances A and B respectively. Blue tunnels indicate pathways which go through neither of the two main entrances described.

Next, tunnels were identified from the substrate binding cavity towards the protein surface. The starting point was defined as the center of mass between the alpha carbon $(\mathrm{C} \alpha)$ atoms of residues 168 and 316 (Figure S7). This starting point was chosen as it is located within the substrate binding cavity and is common to both previously described substrate entrance tunnels (Figure 4). Tunnel frequency and the average bottleneck radius of all tunnels identified in both monomers were obtained for $\mathrm{BZA}_{0}$ and $\mathrm{BZA}_{1}$ (Figure $5 \mathrm{~A}$ and $\mathrm{B}$ ). To aid discussion, we formed electrostatic interactions and instead forming interactions with the solvent in the open conformation; 2) the central region of the gating loop (residues P102, F103, P104) rotates down and away from the hydrophobic core of the entrance cavity. The opening of both entrance A and B involves residues directly interacting with the bilayer. This indicates that the membrane is important in modulating access to the substrate binding pocket of MAO-B.

Evidence of asymmetry in MAO-B from MD simulation. Previous studies have suggested that the presence of an anionic semiquinone could be mechanistically significant as part of a half-site reactivity mechanism. ${ }^{20}$ To investigate the potential for half-site reactivity in MAO-B, tunnels from the $\mathrm{N} 5$ of the flavin to the solvent were first identified from all MD trajectories with $\mathrm{BZA}_{0}, \mathrm{BZA}_{1}$ and $\mathrm{BZA}_{2}$ (with three replicates). These data are useful to determine the size of species that could access the active site and to investigate whether access to the active site is half-site specific. To avoid identifying numerous tunnels that cannot accommodate substrate/product molecules, a minimum tunnel radius was set to $1.5 \AA$. This avoids identifying water tunnels, as water has a Van der Waals radius of $\sim 1.4 \AA .^{63}$ With this criterion, no tunnels were found, which demonstrates a closed active-site on the timescales of our simulations. Whilst larger conformational changes may be occurring on longer time-scales (or in presence of substrate in the vicinity of a bottleneck) to enable substrate access to the active site, a generally closed off active site is in agreement with previous experimental findings. ${ }^{20}$ 


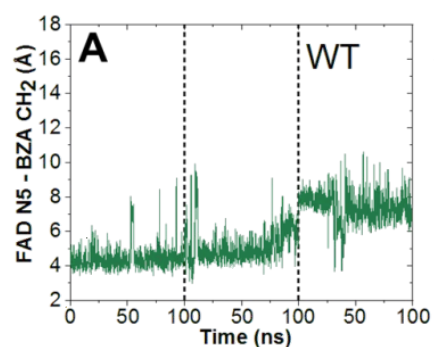

E

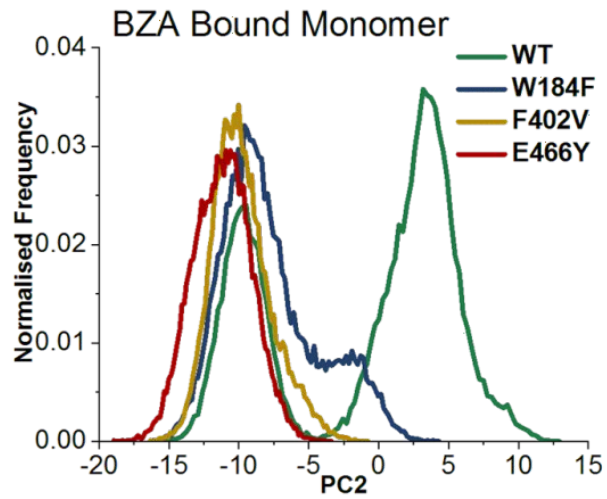

$\mathbf{F}$
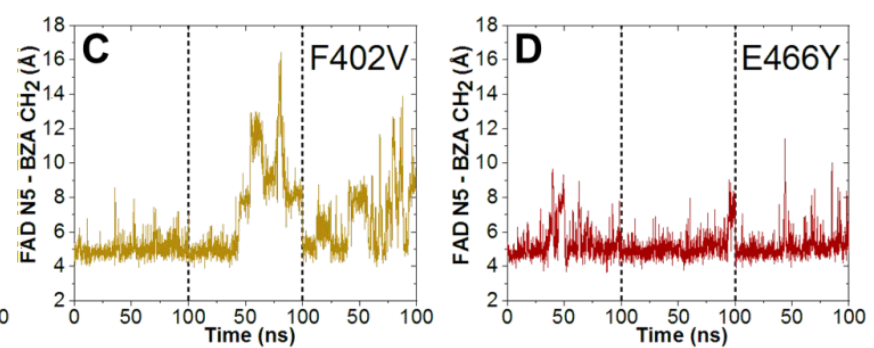

G present in monomer 1 and not monomer 2, the frequency of tunnels and maximum bottleneck measurements are higher for monomer 2 (Figure $5 \mathrm{~A}$ and $\mathrm{B}$ and Table S2). In Figure 5C and $\mathrm{D}$, the pathways are colored according to the entrance pathway they use from the starting point to the solvent, with the majority of tunnels passing the aforementioned entrances $\mathrm{A}$ and $\mathrm{B}$ (Figure 4)

The observed 'closing' of the active site entrance in the $\mathrm{BZA}_{1}$. bound monomer and 'opening' in the $\mathrm{BZA}_{1-\text { unbound }}$ monomer are particularly notable. This indicates that when BZA is bound to the active site cavity of one monomer, a subsequent BZA is more likely to enter the binding site of the opposite monomer. Such asymmetry may prevent binding of subsequent BZA into the same monomer, allowing for the release of products, whilst increasing the efficiency of binding in the opposite 'free' monomer. Previous experimental work indicated that binding of inhibitor to the intermediate binding site of MAO-B was increased where inhibitor was already bound into the active site (see Figure 4 for binding/active site differentiation). ${ }^{64}$ Whilst it is not known whether this is within the same monomer, it could explain the asymmetry in binding site access seen here when $\mathrm{BZA}_{1}$ is present.

$\mathrm{BZA}_{2}$ was not considered for half-site reactivity analysis. This was due to BZA leaving the active site altogether in one simulation trajectory (Figure S8), meaning that the identified tunnels would not be comparable to those where BZA does not leave. This BZA movement may indicate that $\mathrm{BZA}_{2}$ is evidence towards asymmetry in MAO-B.

Mutagenesis reveals long-range networks of motion are important in MAO-B. Our findings above suggest a model where MAO-B intramolecular dynamics are intimately linked to the environment. That is, we hypothesize, that functionally important MAO-B conformational changes are at least in part controlled by the membrane environment. We expect that those conformational changes (or protein dynamics) are influenced by the global protein scaffold. To explore this hypothesis, we have turned to computationally informed mutagenesis studies.

We identify enzyme variants that are predicted to affect networks of flexible motion throughout MAO-B using the FIRST (floppy inclusion and rigid substructure topography) algorithm. ${ }^{65}$ FIRST uses a single conformation (e.g. a crystal structure) to define a constraint-network of movement for a protein. This constraint-network is composed of terms describing covalent and non-covalent contributions to the rigidity of the protein, allowing prediction of the relative rigidity/flexibility of each residue. These calculations can therefore be used to predict the relative rigidity (and therefore stability) of MAO-B variants, both on a local (per-residue) and global (sum of all residues) level.

FIRST was used to perform high-throughput screening to identify point variants that were predicted to significantly rigidify the network of flexible motion of $w t \mathrm{MAO}-\mathrm{B}$, without significantly perturbing the flexibility of residues considered

Figure 6. A-D, Distance between the N5 (FAD) and CH2 group of BZA for wtMAO-B and all three enzyme variant simulations. A black dotted line indicates the start of each new trajectory (all runs performed in triplicate). Additional measurements for all BZA containing simulations can be found in Figures S11 and S12. E + F, Normalized histograms (bin width $0.25 \AA$ ) of principle principal component 2 (PC2) for all BZA1 simulations of the bound (E) and unoccupied monomers (F). G, 'Porcupine' plot of PC2, with arrows indicating the direction of the PC2 eigenvector and arrow size indicating the magnitude of the corresponding eigenvalue, for all $\mathrm{C} \alpha$ atoms with eigenvalues greater than $4 \AA$. The gating loop residues $(99-112)$ are colored in red, and the approximate location of the bilayer is indicated with a black dotted line. (A mobility plot of PC2 is provided in Figure S13A.). 
key for catalysis (see Supporting Information Materials and Methods and Table S3). In short, we perform computational alanine scanning, by applying FIRST with the contribution of each amino-acid side chain to the constraint network discounted in turn. This allows us to identify residues with side chains that make a negligible contribution to the overall rigidity of MAOB. All residues identified with the above approach were then subjected to in silico mutagenesis to all other canonical amino acids and subsequent FIRST analysis.

From these calculations, we selected three enzyme variants (W184F, F402V and E466Y) that, based on our calculations, were predicted to increase the rigidity of the protein scaffold, but are distal to the active site (between 13-21 $\AA$ from BZA) and do not significantly alter the rigidity of catalytically relevant residues (Figure S9 A-C; see SI Materials and Methods). We find that these variants retain the absorption feature at $\sim 415 \mathrm{~nm}$ (Figure S2A) and their overall structure is not significantly perturbed, at least as assessed from their farUV circular dichroism spectra (Figure S3B). However, despite the variants being located a significant distance from the active site, we find that enzyme activity is ablated. Given that these variants are expected to alter the network of flexible motion, we suggest these data reflect the importance of protein conformational changes in MAO-B turnover. Moreover, the importance of such changes may also provide a rationale for the differences of our temperature dependence studies when MAO$\mathrm{B}$ is in different environments (Figure 3).

The FIRST calculations have the advantage that they are rapid and so enable very large in silico screening. However, they are thus necessarily approximate and do not reflect the realistic (and complex) protein molecular dynamics. An additional important caveat of our FIRST calculations is that they do not include the membrane environment. Therefore, in an attempt to rationalize the loss of activity for the variants, and explore our hypothesis above, we have performed 3 independent $100 \mathrm{~ns}$ MD simulations on each variant in the $\mathrm{BZA}_{1}$ state (as above, see $S I$ Materials and Methods).

Based on the flexibility in MD simulations (root-mean square fluctuations, RMSF), each variant is predicted to be slightly more rigid (Figure S9 D-F) than $w t$ MAO-B. Comparison of predictions of relative rigidity (by FIRST) and flexibility (by $\mathrm{MD}$ ) for $w t \mathrm{MAO}-\mathrm{B}$ show only moderate correlation (Figure S10). Poor correlation is particularly found for residues near the bilayer, which is not taken into account in our FIRST calculations; this demonstrates the importance of modelling the molecule in a native-like environment.

Figure 6 A-D, (additional measurements can be found in Figures $\mathrm{S} 11$ and $\mathrm{S} 12$ ) shows the fluctuation in the catalytic distance between BZA and FAD (BZA $\mathrm{CH}_{2}$ and FAD N5) for each variant and $w t \mathrm{MAO}-\mathrm{B}$. There is clearly some fluctuation in individual simulations (with excursions to longer distances), but no significant differences are observed between the variants and $w t$ MAO-B. That is, we do not find any evidence (at least on these timescales) that catalytically competent conformations are disadvantaged in the variants. Therefore, based on these data, we do not expect the immediate active site environment to be compromised in the enzyme variants, which correlates with the observation of the retention of the putative anionic semiquinone peak in the absorption spectrum of each variant (Figure S3).
To explore the changes in global protein dynamics for the MAO-B variants in more detail, we performed principal component analysis (PCA) on the Ca carbon of residues 1-455 for all $\mathrm{BZA}_{1}$ simulations (Figures 6 and S13). The vector which describes each PC can be projected onto a static structure (to create a porcupine plot; Figure $6 \mathrm{G}$ ), with the magnitude of the vector describing the relative change in each residues position over the PC. Analysis of the porcupine plot of PC1 (Figure S13B) shows a global motion dependent on several flexible regions of MAO-B, whereas PC2 is dominated by the movement of the gating loop (residues 99-112) region and residues around it (Figure 6G). This gating loop sits between Entrance A and B (Figure 4) and is key for substrate binding and product release. Histograms of PC2 (Figure 6E and F) show reduced/differential conformational sampling of $\mathrm{PC} 2$ for all variants compared to $w t \mathrm{MAO}-\mathrm{B}$. Our MD simulations therefore suggest that the variants have lost activity because the mutations have altered the normal dynamics of the gating loop, potentially affecting substrate entrance/exit to the active site. The fact that these variants (distal from the gating loop) have such a significant and specific effect on the gating loop sampling implies the presence of a long-range network of motions in MAO-B (Figure 6G) through which dynamics of the gating loop are affected.

\section{Conclusions}

MAO-B is an important biomedical target, and as with many such targets, is associated with a biological membrane. Such association places enzymes in specific physiological contexts, can promote interaction with other species and enable specific structural features. Less obvious is the role of the membrane environment in mediating the conformational dynamics of enzymes, and whether this is functionally important.

Previous molecular dynamics studies have illustrated that the immediate membrane environment of MAO-B is involved in controlling substrate entrance to channels leading to the active site. Here, using enzyme kinetic and mutagenesis studies, we consider the role of the membrane environment in tuning the molecular dynamics of MAO-B more widely, including its influence on turnover and catalysis. We find that placement of MAO-B in SMA nanodiscs instead of in reduced Triton X-100 has a significant effect on the heat capacity of catalysis $\left(\Delta C_{P}^{\ddagger}\right)$. Differences in $\Delta C_{P}^{\ddagger}$ reflect differences in the distribution and frequency of vibrational modes between the ground (reactant) and transition states and implies that the membrane environment is affecting the global protein dynamics of MAO$\mathrm{B}$ and that these dynamics influence the thermodynamics of enzyme turnover. Indeed, $k_{\text {cat }}$ is $\sim 5$ times faster in the nanodisc environment versus reduced Triton X-100. These data further hint at the role of the specific lipid composition and fine structure of the membrane to tune MAO-B turnover efficiency. These findings are corroborated by studying enzyme variants that are predicted to alter the network of flexible motion in the enzyme, but do not affect the overall structure. These variants, which are distal to the active site, all lead to inactive enzyme, indicating the critical role of 'optimized' global protein dynamics of MAO-B.

Through atomistic molecular dynamics simulations with and without substrate bound, we find two substrate entrance/product exit channels that are mediated by membrane interaction, one 
of which was not previously described. The simulations further indicate an asymmetry in substrate access in the MOAB dimer. Specifically, either one or the other monomer may allow substrate access at any one time, with active site occupation in one monomer preferentially allowing substrate access to the other. Moreover, the gating loop dynamics appear to be highly sensitive to the global enzyme dynamics, potentially reflecting long-range networks of enzyme motion.

Taken together, our study suggests that the global protein dynamics of MAO-B are 'tuned' by the specific immediate membrane environment. These protein dynamics have a major effect on MAO-B function, through tuning fluctuations linked to enzyme turnover, including controlling the opening and closing of substrate/product channels. The finding that two different channels mediated by the membrane environment are present in MAO-B illustrates the potential to exploit novel small molecule binding sites with rational drug design. Therefore, our study illustrates that when searching for novel small molecule binding sites, one should consider not just the static structure of the system in isolation, but time-dependent changes in the population of conformational sub-states and in the 'native' environment. ${ }^{66,67}$ The finding that there may be long range networks of motions that can, in particular, affect the gating loop also indicates the potential for allosteric inhibitors. Further, as MAO-B resides in the outer mitochondrial membrane of cells, the finding of a solvent accessible entrance is important for rational drug design efforts. That is, inhibitors that target MAO-B may not need to enter the mitochondrial membrane in order to access the active site of MAO-B.

\section{ASSOCIATED CONTENT}

Supporting Information. FIRST calculations methodology, molecular dynamics simulation methodology, MAO-B mechanisms, table of caver data, $\mathrm{MAO}-\mathrm{B}$ variant and environment spectra, Michealis-Menten of Y398F MAO-B, MD RMSD and area per lipid calculations, Entrance B structure, Caver calculation starting point, RMSF of gating loop, BZA leading the active site by MD simulations, Michaelis-Mentens of $w t$ MAO-B. This information is available free of charge on the ACS Publications website.

Protein Accession ID. GLCDH_SACSO 093715

\section{AUTHOR INFORMATION}

\section{Corresponding Authors}

*Christopher R Pudney, Department of Biology and Biochemistry, University of Bath, Bath, United Kingdom. c.r.pudney@bath.ac.uk.

* Marc W van der Kamp, School of Biochemistry, University of Bristol, Cantock's Close, Bristol BS8 1TS, United Kingdom. marc.vanderkamp@bristol.ac.uk.

\section{Author Contributions}

HBLJ and AM performed experimental work. RMC and HBLJ performed computational calculations and simulations. All authors discussed and interpreted data. The manuscript was written through contributions of all authors. All authors have given approval to the final version of the manuscript. HBLJ and RMC contributed equally"l.

\section{ABBREVIATIONS}

Alpha carbon $(\mathrm{C} \alpha)$, benzylamine (BZA), electron paramagnetic resonance (EPR), flavin adenine dinucleotide (FAD), heat capacity of catalysis $\left(\Delta C_{P}^{\ddagger}\right)$, kynuramine $(\mathrm{KYN})$, molecular dynamics (MD), monoamine oxidase (MAO), principal component analysis (PCA), palmitoyloleoylphosphatidylcholine (POPC), palmitoyloleoyl-phosphatidylethanolamine (POPE), root mean square fluctuation (RMSF), reactive oxygen species (ROS).

\section{ACKNOWLEDGMENT}

HBLJ's studentship is funded by the University of Bath. RMC's studentship is funded by the EPSRC. MWvdK is a BBSRC David Phillips Fellow (BB/M026280/1). SAW has received funding from the European Research Council (ERC) under the European Union's Horizon 2020 research and innovation programme (grant agreement No 648283 "GROWMOF"). This research made use of the Balena High Performance Computing (HPC) Service at the University of Bath

\section{REFERENCES}

(1) Binda, C.; Newton-Vinson, P.; Hubálek, F.; Edmondson, D. E.; Mattevi, A. Structure of Human Monoamine Oxidase B, a Drug Target for the Treatment of Neurological Disorders. Nat. Struct. Biol. 2002, 9, 22-26.

(2) Schnaitman, C.; Erwin, V. G.; Greenawalt, J. W. The Submitochondrial Localization of Monoamine Oxidase. An Enzymatic Marker for the Outer Membrane of Rat Liver Mitochondria. J. Cell Biol. 1967, 32, 719-735.

(3) Knoll, J.; Ecseri, Z.; Kelemen, K.; Nievel, J.; Knoll, B. Phenylisopropylmethylpropinylamine (E-250), a New Spectrum Psychic Energizer. Arch. Int. Pharmacodyn. Ther. 1965, 155, 154-164.

(4) Riederer, P.; Laux, G. MAO-Inhibitors in Parkinson's Disease. Exp. Neurobiol. 2011, 20, 1-17.

(5) Jonsson, T.; Edmondson, D. E.; Klinman, J. P. Hydrogen Tunneling in the Flavoenzyme Monoamine Oxidase B. Biochemistry 1994, $33,14871-14878$

6) Nagel, Z. D.; Klinman, J. P. Tunneling and Dynamics in Enzymatic Hydride Transfer. Chem. Rev. 2006, 106, 3095-3118.

(7) MacMillar, S.; Edmondson, D. E.; Matsson, O. Nitrogen Kinetic Isotope Effects for the Monoamine Oxidase B-Catalyzed Oxidation of Benzylamine and (1,1- 2 H 2 )Benzylamine: Nitrogen Rehybridization and CH Bond Cleavage Are Not Concerted. J. Am. Chem. Soc. 2011, 133, 12319-12321.

(8) Silverman, R. B. Radical Ideas about Monoamine Oxidase. Acc. Chem. Res. 1995, 28, 335-342.

(9) Akyüz, M. A.; Erdem, S. S. Computational Modeling of the Direct Hydride Transfer Mechanism for the MAO Catalyzed Oxidation of Phenethylamine and Benzylamine: ONIOM (QM/QM) Calculations. J. Neural Transm. 2013, 120, 937-945.

(10) Kurtz, K. A.; Rishavy, M. A.; Cleland, W. W.; Fitzpatrick, P. F. Nitrogen Isotope Effects as Probes of the Mechanism of D-Amino Acid Oxidase. J. Am. Chem. Soc. 2000, 122, 12896-12897.

(11) Fitzpatrick, P. F. Oxidation of Amines by Flavoproteins. Arch. Biochem. Biophys. 2010, 493, 13-25.

(12) Vianello, R.; Repič, M.; Mavri, J. How Are Biogenic Amines Metabolized by Monoamine Oxidases? European J. Org. Chem. 2012 2012, 7057-7065.

(13) Walker, M. C.; Edmondson, D. E. Structure-Activity Relationships in the Oxidation of Benzylamine Analogs by Bovine Liver Mitochondrial Monoamine Oxidase B. Biochemistry 1994, 33, 7088-7098.

(14) Abad, E.; Zenn, R. K.; Kästner, J. Reaction Mechanism of Monoamine Oxidase from QM/MM Calculations. J. Phys. Chem. B 2013, $117,14238-14246$ 
(15) Murray, A. T.; Dowley, M. J. H.; Pradaux-Caggiano, F.; Baldansuren, A.; Fielding, A. J.; Tuna, F.; Hendon, C. H.; Walsh, A.; LloydJones, G. C.; John, M. P.; Carbery, D. R. Catalytic Amine Oxidation under Ambient Aerobic Conditions: Mimicry of Monoamine Oxidase B. Angew. Chemie - Int. Ed. 2015, 54, 8997-9000.

(16) Rigby, S. E. J.; Hynson, R. M. G.; Ramsay, R. R.; Munro, A. W.; Scrutton, N. S. A Stable Tyrosyl Radical in Monoamine Oxidase A. J. Biol. Chem. 2005, 280, 4627-4631.

(17) Dunn, R. V.; Munro, A. W.; Turner, N. J.; Rigby, S. E. J.; Scrutton, N. S. Tyrosyl Radical Formation and Propagation in Flavin Dependent Monoamine Oxidases. ChemBioChem 2010, 11, 1228-1231.

(18) Newton-Vinson, P.; Hubalek, F.; Edmondson, D. E. High-Level Expression of Human Liver Monoamine Oxidase B in Pichia Pastoris. Protein Expr. Purif. 2000, 20, 334-345.

(19) Yue, K. T.; Bhattacharyya, A. K.; Zhelyaskov, V. R.; Edmondson, D. E. Resonance Raman Spectroscopic Evidence for an Anionic Flavin Semiquinone in Bovine Liver Monoamine Oxidase. Arch. Biochem. Biophys. 1993, 300, 178-185.

(20) DeRose, V. J.; Woo, J. C. G.; Hawe, W. P.; Hoffman, B. M.; Silverman, R. B.; Yelekci, K. Observation of a Flavin Semiquinone in the Resting State of Monoamine Oxidase B by Electron Paramagnetic Resonance and Electron Nuclear Double Resonance Spectroscopy. Biochemistry 1996, 35, 11085-11091.

(21) Allen, W. J.; Bevan, D. R. Steered Molecular Dynamics Simulations Reveal Important Mechanisms in Reversible Monoamine Oxidase B Inhibition. Biochemistry 2011, 50, 6441-6454.

(22) Is, Y. S.; Durdagi, S.; Aksoydan, B.; Yurtsever, M. Proposing Novel MAO-B Hit Inhibitors Using Multidimensional Molecular Modeling Approaches and Application of Binary QSAR Models for Prediction of Their Therapeutic Activity, Pharmacokinetic and Toxicity Properties. ACS Chem. Neurosci. 2018, 9, 1768-1782.

(23) Braun, G. H.; M Jorge, D. M.; Ramos, H. P.; Alves, R. M.; da Silva, V. B.; Giuliatti, S.; Vilela Sampaio, S.; Taft, C. A.; T P Silva, C. H. Molecular Dynamics, Flexible Docking, Virtual Screening, ADMET Predictions, and Molecular Interaction Field Studies to Design Novel Potential MAO-B Inhibitors. J. Biomol. Struct. Dyn. 2008, 254, 347-355.

(24) La Regina, G.; Silvestri, R.; Artico, M.; Lavecchia, A.; Novellino, E.; Befani, O.; Turini, P.; Agostinelli, E. New Pyrrole Inhibitors of Monoamine Oxidase: Synthesis, Biological Evaluation, and Structural Determinants of MAO-A and MAO-B Selectivity. J. Med. Chem. 2007, 50, 922-931.

(25) Maccioni, E.; Alcaro, S.; Cirilli, R.; Vigo, S.; Cardia, M. C.; Sanna, M. L.; Meleddu, R.; Yanez, M.; Costa, G.; Casu, L.; Matyus, P.; Distinto, S. 3-Acetyl-2,5-Diaryl-2,3-Dihydro-1,3,4-Oxadiazoles: A New Scaffold for the Selective Inhibition of Monoamine Oxidase B. J. Med. Chem. 2011, 54, 6394-6398.

(26) Chimenti, F.; Maccioni, E.; Secci, D.; Bolasco, A.; Chimenti, P.; Granese, A.; Befani, O.; Turini, P.; Alcaro, S.; Ortuso, F.; Cirilli, R.; Torre, F. La; Cardia, M. C.; Distinto, S. Synthesis, Molecular Modeling Studies, and Selective Inhibitory Activity against Monoamine Oxidase of 1Thiocarbamoyl-3,5-Diaryl-4,5-Dihydro-(1H)- Pyrazole Derivatives. J. Med. Chem. 2005, 48, 7113-7122.

(27) Fowler, P. W.; Balali-Mood, K.; Deol, S.; Coveney, P. V.; Sansom, M. S. P. Monotopic Enzymes and Lipid Bilayers: A Comparative Study. Biochemistry 2007, 46, 3108-3115.

(28) Evans, E. W.; Dodson, C. A.; Maeda, K.; Biskup, T.; Wedge, C. J.; Timme, C. R. Magnetic Field Effects in Flavoproteins and Related Systems. Interface Focus 2013, 3.

(29) McDowall, J. S.; Ntai, I.; Hake, J.; Whitley, P. R.; Mason, J. M.; Pudney, C. R.; Brown, D. R. Steady-State Kinetics of $\alpha$-Synuclein Ferrireductase Activity Identifies the Catalytically Competent Species. Biochemistry 2017, 56, 2497-2505.

(30) Weyler, W.; Salach, J. I. Purification and Properties of Mitochondrial Monoamine Oxidase Type A from Human Placenta. J. Biol. Chem. 1985, 260, 13199-13207.
(31) Stoll, S.; Schweiger, A. EasySpin, a Comprehensive Software Package for Spectral Simulation and Analysis in EPR. J. Magn. Reson. 2006, 178, 42-55.

(32) Binda, C.; Hubálek, F.; Li, M.; Herzig, Y.; Sterling, J.; Edmondson, D. E.; Mattevi, A. Crystal Structures of Monoamine Oxidase B in Complex with Four Inhibitors of the N-Propargylaminoindan Class. J. Med. Chem. 2004, 47, 1767-1774.

(33) Hanwell, M. D.; Curtis, D. E.; Lonie, D. C.; Vandermeersch, T.; Zurek, E.; Hutchison, G. R. Avogadro: An Advanced Semantic Chemical Editor, Visualization, and Analysis Platform. J. Cheminform. 2012, 4, 17.

(34) Wu, E. L.; Cheng, X.; Jo, S.; Rui, H.; Song, K. C.; DávilaContreras, E. M.; Qi, Y.; Lee, J.; Monje-Galvan, V.; Venable, R. M.; Klauda, J. B.; Im, W. CHARMM-GUI Membrane Builder toward Realistic Biological Membrane Simulations. J. Comput. Chem. 2014, 35, $1997-$ 2004.

(35) van Meer, G.; Voelker, D. R.; Feigenson, G. W. Membrane Lipids: Where They Are and How They Behave. Nat. Rev. Mol. Cell Biol. $2008,9,112-124$.

(36) Li, M.; Binda, C.; Mattevi, A.; Edmondson, D. E. Functional Role of The "aromatic Cage" in Human Monoamine Oxidase B: Structures and Catalytic Properties of Tyr435 Mutant Proteins. Biochemistry 2006, 45, $4775-4784$.

(37) Edmondson, D. E.; Binda, C.; Wang, J.; Upadhyay, A. K.; Mattevi, A. Molecular and Mechanistic Properties of the Membrane-Bound Mitochondrial Monoamine Oxidases. Biochemistry 2009, 48, 4220-4230.

(38) Søndergaard, C. R.; Olsson, M. H. M.; Rostkowski, M.; Jensen, J. H. Improved Treatment of Ligands and Coupling Effects in Empirical Calculation and Rationalization of P K a Values. J. Chem. Theory Comput. 2011, 7, 2284-2295.

(39) Chen, V. B.; Arendall, W. B.; Headd, J. J.; Keedy, D. A.; Immormino, R. M.; Kapral, G. J.; Murray, L. W.; Richardson, J. S.; Richardson, D. C. MolProbity: All-Atom Structure Validation for Macromolecular Crystallography. Acta Crystallogr. Sect. D Biol. Crystallogr. 2010, 66, 12-21.

(40) Best, R. B.; Zhu, X.; Shim, J.; Lopes, P. E. M.; Mittal, J.; Feig, M.; MacKerell, A. D. Optimization of the Additive CHARMM All-Atom Protein Force Field Targeting Improved Sampling of the Backbone $\Phi, \psi$ and Side-Chain $\chi 1$ and $\chi 2$ Dihedral Angles. J. Chem. Theory Comput. 2012, $8,3257-3273$.

(41) Klauda, J. B.; Venable, R. M.; Freites, J. A.; O’Connor, J. W.; Tobias, D. J.; Mondragon-Ramirez, C.; Vorobyov, I.; MacKerell, A. D.; Pastor, R. W. Update of the CHARMM All-Atom Additive Force Field for Lipids: Validation on Six Lipid Types. J. Phys. Chem. B 2010, 114, 78307843.

(42) Darden, T.; York, D.; Pedersen, L. Particle Mesh Ewald: An N $\cdot \log (\mathrm{N})$ Method for Ewald Sums in Large Systems. J. Chem. Phys. 1993 , 98, 10089-10092.

(43) Roe, D. R.; Cheatham, T. E. PTRAJ and CPPTRAJ: Software for Processing and Analysis of Molecular Dynamics Trajectory Data. J. Chem. Theory Comput. 2013, 9, 3084-3095.

(44) Allen, W. J.; Lemkul, J. A.; Bevan, D. R. GridMAT-MD: A Grid-Based Membrane Analysis Tool for Use with Molecular Dynamics. J. Comput. Chem. 2009, 30, 1952-1958.

(45) Chovancova, E.; Pavelka, A.; Benes, P.; Strnad, O.; Brezovsky, J.; Kozlikova, B.; Gora, A.; Sustr, V.; Klvana, M.; Medek, P.; Biedermannova, L.; Sochor, J.; Damborsky, J. CAVER 3.0: A Tool for the Analysis of Transport Pathways in Dynamic Protein Structures. PLoS Comput. Biol. 2012, 8, e1002708.

(46) Perkins, D. N.; Pappin, D. J. C.; Creasy, D. M.; Cottrell, J. S. Probability-Based Protein Identification by Searching Sequence Databases Using Mass Spectrometry Data. Electrophoresis 1999, 20, 3551-3567.

(47) Kao, Y. T.; Saxena, C.; He, T. F.; Guo, L.; Wang, L.; Sancar, A.; Zhong, D. Ultrafast Dynamics of Flavins in Five Redox States. J. Am. Chem. Soc. 2008, 130, 13132-13139. 
(48) Candeias, L. P.; Turconi, S.; Nugent, J. H. A. Tyrosine Y(Z) and Y(D) of Photosystem II Comparison of Optical Spectra to Those of Tyrosine Oxidised by Pulsed Radiolysis. Biochim. Biophys. Acta Bioenerg. 1998, 1363, 1-5.

(49) Geha, R. M.; Chen, K.; Wouters, J.; Ooms, F.; Shih, J. C. Analysis of Conserved Active Site Residues in Monoamine Oxidase A and B and Their Three-Dimensional Molecular Modeling. J. Biol. Chem. 2002, 277, 17209-17216.

(50) Woo, J. C. G.; Silverman, R. B. Observation of Two Different Chromophores in the Resting State of Monoamine Oxidase B by Fluorescence Spectroscopy. Biochem. Biophys. Res. Commun. 1994, 202, $1574-1578$

(51) Aubert, C.; Brettel, K.; Mathis, P.; Eker, A. P. M.; Boussac, A. EPR Detection of the Transient Tyrosyl Radical in DNA Photolyase from Anacystis Nidulans. J. Am. Chem. Soc. 1999, 121, 8659-8660.

(52) Okafuji, A.; Schnegg, A.; Schleicher, E.; Möbius, K.; Weber, S. G-Tensors of the Flavin Adenine Dinucleotide Radicals in Glucose Oxidase: A Comparative Multifrequency Electron Paramagnetic Resonance and Electron-Nuclear Double Resonance Study. J. Phys. Chem. B 2008, 112, 3568-3574.

(53) Hobbs, J. K.; Jiao, W.; Easter, A. D.; Parker, E. J.; Schipper, L. a.; Arcus, V. L. Change in Heat Capacity for Enzyme Catalysis Determines Temperature Dependence of Enzyme Catalyzed Rates. ACS Chem. Biol. 2013, 8, 2388-2393.

(54) Jones, H. B.; Wells, S. A.; Prentice, E. J.; Kwok, A.; Liang, L. L.; Arcus, V. L.; Pudney, C. R. A Complete Thermodynamic Analysis of Enzyme Turnover Links the Free Energy Landscape to Enzyme Catalysis. FEBS J. 2017, 284, 2829-2842.

(55) van der Kamp, M. W.; Prentice, E. J.; Kraakman, K. L.; Connolly, M.; Mulholland, A. J.; Arcus, V. L. Dynamical Origins of Heat Capacity Changes in Enzyme-Catalysed Reactions. Nat. Commun. 2018, 9 , 1177.

(56) Jones, H. B. L.; Crean, R. M.; Matthews, C.; Troya, A. B.; Danson, M. J.; Bull, S. D.; Arcus, V. L.; van der Kamp, M. W.; Pudney, C. R. Uncovering the Relationship between the Change in Heat Capacity for Enzyme Catalysis and Vibrational Frequency through Isotope Effect Studies. ACS Catal. 2018, 8, 5340-5349.

(57) Longbotham, J. E.; Hardman, S. J. O.; Gö, S.; Scrutton, N. S.; Hay, S. Untangling Heavy Protein and Cofactor Isotope Effects on EnzymeCatalyzed Hydride Transfer. J. Am. Chem. Soc 2016, 138, 13693-13699.

(58) Arcus, V. L.; Prentice, E. J.; Hobbs, J. K.; Mulholland, A. J.; van der Kamp, M. W.; Pudney, C. R.; Parker, E. J.; Schipper, L. A. On the Temperature Dependence of Enzyme-Catalyzed Rates. Biochemistry 2016, $55,1681-1688$.

(59) Pavelka, A.; Sebestova, E.; Kozlikova, B.; Brezovsky, J.; Sochor, J.; Damborsky, J. CAVER: Algorithms for Analyzing Dynamics of Tunnels in Macromolecules. IEEE/ACM Trans. Comput. Biol. Bioinforma. 2016, 13, 505-517.

(60) Kingsley, L. J.; Lill, M. A. Including Ligand-Induced Protein Flexibility into Protein Tunnel Prediction. J. Comput. Chem. 2014, 35, $1748-1756$.

(61) Biedermannová, L.; Prokop, Z.; Gora, A.; Chovancová, E.; Kovács, M.; Damborský, J.; Wade, R. C. A Single Mutation in a Tunnel to the Active Site Changes the Mechanism and Kinetics of Product Release in Haloalkane Dehalogenase LinB. J. Biol. Chem. 2012, 287, 29062-29074.

(62) Zapata-Torres, G.; Fierro, A.; Miranda-Rojas, S.; Guajardo, C.; Saez-Briones, P.; Salgado, J. C.; Celis-Barros, C. Influence of Protonation on Substrate and Inhibitor Interactions at the Active Site of Human Monoamine Oxidase-A. J. Chem. Inf. Model. 2012, 52, 1213-1221.

(63) Tabbutt, F. Water: A Matrix of Life, 2nd Edition (Franks, Felix). J. Chem. Educ. 2001, 78, 593.

(64) Bonivento, D.; Milczek, E. M.; McDonald, G. R.; Binda, C.; Holt, A.; Edmondson, D. E.; Mattevi, A. Potentiation of Ligand Binding through Cooperative Effects in Monoamine Oxidase B. J. Biol. Chem. 2010, 285, 36849-36856.
(65) Wells, S.; Menor, S.; Hespenheide, B.; Thorpe, M. F. Constrained Geometric Simulation of Diffusive Motion in Proteins. Phys. Biol. 2005, 2.

(66) Oleinikovas, V.; Saladino, G.; Cossins, B. P.; Gervasio, F. L. Understanding Cryptic Pocket Formation in Protein Targets by Enhanced Sampling Simulations. J. Am. Chem. Soc. 2016, 138, 14257-14263.

(67) Beglov, D.; Hall, D. R.; Wakefield, A. E.; Luo, L.; Allen, K. N.; Kozakov, D.; Whitty, A.; Vajda, S. Exploring the Structural Origins of Cryptic Sites on Proteins. Proc. Natl. Acad. Sci. U. S. A. 2018, 115, 34163425. 
2

3

4

5

6

7

8

9

10

11

12

13

14

15

16

17

18

19

20

21

22

23

24

25

27

28

29

30

31

32

33

34

35

36

37

38

39

40

41

42

43

44

45

46

47

48

49

50

51

52

53

54

55

56

57

58

59

60

ACS Paragon Plus Environment 


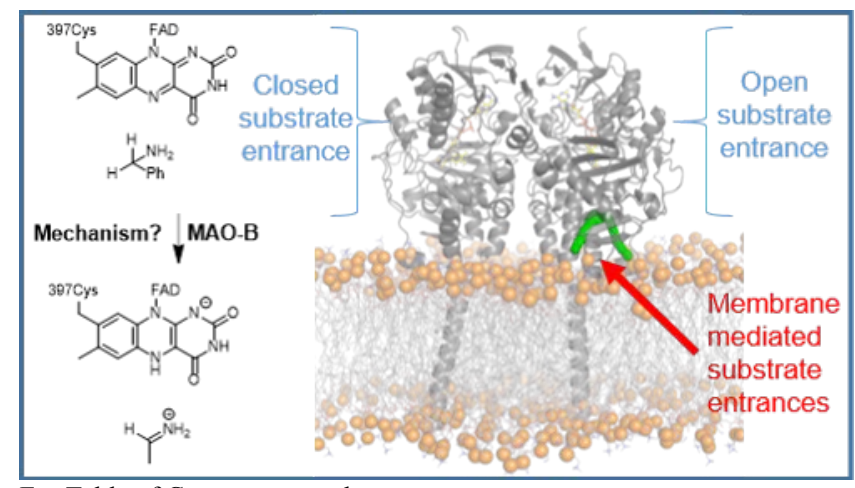

For Table of Contents use only 